\title{
Pensamento matemático avançado manifestado em tarefas envolvendo transformações lineares
}

\author{
Advanced mathematical thinking in tasks \\ involving linear transformations
}

Alessandra Senes Marins ${ }^{1}$ • Angela Marta Pereira das Dores Savioli ${ }^{1}$

\begin{abstract}
Resumo: Esse artigo apresenta os resultados de uma pesquisa de mestrado cujo objetivo foi identificar e discutir que indícios/características de processos do Pensamento Matemático Avançado estudantes de um curso de Matemática manifestam ao lidarem com tarefas referentes ao conteúdo de transformações lineares. Para isso, realizou-se um estudo a respeito de algumas teorias do Pensamento Matemático Avançado, o qual serviu de base para analisar os registros escritos dos estudantes. Concluiu-se que alguns participantes da pesquisa manifestaram características dos processos de representação e abstração do Pensamento Matemático Avançado, sendo que apenas dois apresentaram indícios dos processos envolvidos na abstração. Os registros escritos desses estudantes apresentam dificuldades com as notações para os conceitos em questão, assim como com a manipulação dessas notações. Assim, existe a necessidade dos professores oportunizarem momentos de reflexão em relação aos objetos dessa disciplina, como o desenvolvimento do Pensamento Matemático Avançado.
\end{abstract}

Palavras-chave: Educação matemática. Pensamento matemático avançado. Álgebra linear. Ensino superior.

\begin{abstract}
This article presents the results of research that aimed to identify and discuss the evidence and characteristics of the processes of the Advanced Mathematical Thinking students in a mathematics course expressed when dealing with tasks related to the content of linear transformations. For this, we designed a study using some theories of Advanced Mathematical Thinking, which served as the foundation for analyzing students' written records. We conclude that some participants of the research expressed characteristics of the processes of representation and abstraction of Advanced Mathematical Thinking, and only two showed evidence of processes involved in abstraction. These students' written records present difficulties with notation for the concepts in question, as with the manipulation of these notations. Thus, there is a need for professors to create moments of reflection in relation to the development of Advanced Mathematical Thinking.
\end{abstract}

Keywords: Mathematics education. Advanced mathematical thinking. Linear algebra. Higher education.

\footnotetext{
${ }^{1}$ Universidade Estadual de Londrina (UEL), Programa de Pós-Graduação em Ensino de Ciências e Educação Matemática, Londrina, PR, Brasil.E-mail: <ale_marins@hotmail.com>
} 


\section{Introdução}

O ensino da matemática, mais especificamente da álgebra, nos diferentes níveis de escolaridade, tem sido foco de estudo de pesquisadores e professores que buscam novas abordagens para a sala de aula, pois, em geral, a matemática é ensinada mecanicamente, enfatizando procedimentos de memorização, de reprodução de algoritmos, sem proporcionar aos estudantes a compreensão dos significados de objetos matemáticos, como citam Fiorentini, Miorin e Miguel (1993, p. 40) a respeito do ensino da álgebra: "[...] a maioria dos professores ainda trabalha a Álgebra - de forma mecânica e automatizada, dissociada de qualquer significação social e lógica, enfatizando simplesmente a memorização e a manipulação de regras, macetes, símbolos e expressões - tal como ocorria há várias décadas".

Essa forma de ensino por meio de manipulação de símbolos e repetição de técnicas não fica restrita à Educação Básica, ultrapassa suas barreiras e estende-se ao Ensino Superior, pois é comum no próprio curso de Matemática o ensino por meio da reprodução de procedimentos.

De acordo com Tall (2002), o ensino da matemática no Ensino Superior, na maioria das vezes, ao invés de promover o estudante a participar de um ciclo criativo (no qual o estudante considera o contexto de um problema em investigação que o conduz à formulação de conjecturas a fim de levá-lo ao refinamento e à prova do processo de construção de um objeto matemático), inicia-se com a forma final da teoria deduzida, ou seja, o produto do Pensamento Matemático Avançado (PMA).

Dreyfus (2002) afirma que os estudantes vêm sendo ensinados a partir do produto da atividade de matemáticos em sua forma final ao invés de serem conduzidos a processos que levaram os matemáticos a construírem esses produtos. De acordo o autor, para um estudante atingir a compreensão de um objeto matemático não é suficiente apenas definir e exemplificar um conceito abstrato, mas sim construir as propriedades de um determinado conceito por meio de deduções a partir da definição. A reflexão em relação à própria experiência matemática é um aspecto importante para o aprendizado de um conceito matemático e uma característica do PMA.

Além disso, outros aspectos de um objeto matemático, que antecedem o produto final, como a notação e a manipulação dos símbolos presentes em questão, comumente são realizados sem a compreensão dos seus significados. Estudantes no Ensino Superior apresentam dificuldades com essas notações e a sua manipulação, pois, além de uma gama de símbolos que não eram comuns durante a Educação Básica, trazem consigo elementos matemáticos a serem compreendidos antes da realização da manipulação.

Uma das disciplinas que exige um formalismo por parte dos estudantes, com o qual não estão acostumados, é a de Álgebra Linear, a qual é ministrada nos primeiros anos do curso de matemática. Esse é um dos motivos pelo qual existe a necessidade dos professores oportunizarem momentos de reflexão em relação aos objetos dessa disciplina, afinal apenas definir e exemplificar conceitos/objetos matemáticos abstratos promovendo a repetição de procedimentos e técnicas não são suficientes para a sua compreensão.

Sendo assim, a Álgebra Linear desempenha um papel importante no ensino da Matemática, pois

[...] conduz a muitos ramos da matemática com os quais tem ligações, incluindo álgebra abstrata, matemática discreta, cálculo, equações diferenciais, 
geometria, estatística, métodos numéricos, pesquisa operacional e sistemas dinâmicos. Ela expõe os estudantes a aspectos teóricos, aspectos aplicados e aspectos numéricos da matemática e tem aplicações em uma gama variada de disciplinas. (POOLE, 2004, p. xii)

Diante da sua importância para a própria matemática e outras áreas, escolhemos o conteúdo de transformações lineares, o qual faz parte da disciplina de Álgebra Linear, para realizarmos nossa investigação a fim de identificar e discutir que indícios/características de processos do Pensamento Matemático Avançado são manifestados por estudantes do curso de Matemática de uma universidade do norte do Paraná ao lidarem com tarefas referentes ao conteúdo de transformações lineares.

\section{Pensamento Matemático Avançado}

Dreyfus (2002), Tall (1995) e Resnick (1987) compartilham de algumas ideias em comum para caracterização do PMA seguindo uma perspectiva cognitivista. Por esse motivo, realizamos um estudo comparativo entre esses autores e optamos por utilizar como base os processos de representação e abstração do PMA segundo a teoria de Dreyfus (2002) e anexar as semelhanças segundo Resnick (1987) e Tall (1995) nesses processos.

Comparando os processos do Pensamento Matemático Avançado de Dreyfus (2002) com as características que Resnick (1987) aponta para o Pensamento de Ordem Superior ${ }^{2}$, listamos algumas semelhanças. De acordo com a autora, esse tipo de pensamento não é algoritmo, ou seja, não existe uma sequência pré-estabelecida pela qual o professor delimite os passos e a partir disso o estudante consiga chegar à abstração do conceito envolvido.

Para Dreyfus (2002), muitas vezes, em sala de aula isso acontece porque os professores constroem sequências, sínteses, a fim de facilitar a compreensão de seus estudantes. Para o autor, apesar da intenção do professor ser boa, o processo de construção não foi realizado pelo estudante e, consequentemente, as possíveis reflexões e os processos de representação e de abstração não foram realizados por seus alunos, além disso, muitas vezes os processos utilizados por um indivíduo não são os mesmos usados por outro. Desse modo, vemos que os processos do PMA não podem ser considerados como um algoritmo, ou seja, suas ações não são previstas, e, portanto, o processo não é conhecido (RESNICK, 1987).

A resolução de um problema possibilita o desenvolvimento dos processos do PMA dos estudantes, além de que contribui para o desenvolvimento de habilidades e competências. Afinal, quando um indivíduo estabelece várias representações para abstrair um conceito, ele precisa gerenciá-las de modo que estabeleça relações "fortes" entre elas, integrando-as e, sempre que necessário, fazendo a mudança entre as representações para atingir seu objetivo (DREYFUS, 2002).

\footnotetext{
${ }^{2}$ Para Resnick (1987) o "Pensamento Avançado" não é exclusivo da área de matemática e por esse motivo a autora o nomeia como Pensamento de Ordem Superior.
} 
O processo de gerenciamento das representações envolve a "auto-regulação" de sua aprendizagem, envolve "julgamento e interpretação com nuances”, características descritas por Resnick (1987) para o Pensamento de Ordem Superior. Além disso, as várias representações existentes, mesmo que pareçam desconexas, tornam-se fortemente ligadas, "envolvendo um significado abrangente", resultando em uma estrutura que aparentemente estava em desordem (RESNICK, 1987). Para Dreyfus (2002) a "auto-regulação" é a parte fascinante do processo de representação do PMA, pois "[...] a pessoa tem o controle sobre as representações que se quer usar” (DREYFUS, 2002, p. 39, tradução nossa).

E, por fim, segundo as características de Resnick (1987, p.3, tradução nossa) a respeito do Pensamento de Ordem Superior, a autora afirma que esse "[...] exige esforço. Há um trabalho mental considerável envolvido nas elaborações e nos julgamentos exigidos"; e, de acordo com Dreyfus (2002), a realização da abstração por parte de um estudante é uma atividade de construção mental a qual depende da atenção centrada por ele nas estruturas que formarão parte do conceito abstrato.

Para Tall (1995), o PMA inicia-se com o desenvolvimento do Pensamento Matemático Elementar que, em geral, depende mais das situações manipuláveis, das sensações físicas, mas para se tornar avançado começa a depender menos dessas sensações e mais das construções internas. Desse modo, os objetos passam de visuais-espaciais, para, sucessivamente, mais verbais-dedutivos.

Para o autor, um conceito ou objeto matemático utiliza de símbolos, como imagem, para ligar o processo apropriado e suas relações na estrutura cognitiva, ou seja, existe um esquema mental entrelaçado a esse símbolo que representa o objeto. Por isso, quando nos deparamos com um objeto matemático, buscamos em nossa mente símbolos possíveis de manipular como se fossem objetos mentais para a representação do objeto. Comparando com a caracterização de Dreyfus (2002) para o PMA, podemos inferir que um processo de representação mental, quando utiliza de símbolos ou da visualização para gerar esquemas mentais do conceito ou objeto, é semelhante à caracterização de Tall (1995) para o objeto mental.

De acordo com Tall (1995), quando as ações tornam-se simbolizadas como processos, encapsuladas como proceitos (processo ocorrido para entender um conceito), houve a mudança da fase cognitiva do Pensamento Matemático Elementar para o Avançado, pois o objeto matemático tornou-se compreendido, "encapsulado", passando a ter um novo status cognitivo: da descrição para a definição, ou seja, houve “[...] a transição da coerência da matemática elementar para a consequência da matemática avançada, baseada em entidades abstractas que o indivíduo deve construir através de deduções das definições formais" (DOMINGOS, 2006, p. 5).

Sendo assim, em Dreyfus (2002) entendemos que essa passagem é a abstração, ou seja, os processos do PMA foram utilizados com a finalidade de compreender o objeto, como um todo, para que suas relações e propriedades sejam abstraídas. Dessa forma, os processos que estão envolvidos nessa parte são o da generalização e da síntese.

Com o objetivo de buscar as semelhanças de características do PMA entre a concepção de Dreyfus (2002) em relação à de Tall (1995) e à de Resnick (1987), construímos um quadro que retrata a síntese dessas semelhanças. Esse quadro foi construído tomando como base os processos de representação e abstração do PMA segundo Dreyfus (2002) relacionando-os com algumas características de Tall (1995) e Resnick (1987). Para isso, cada processo do PMA foi codificado com uma sigla com o intuito de facilitar a construção dos quadros referentes às análises dos indícios e características do PMA. 
Para a análise dos indícios/características dos processos do PMA, manifestados nos registros escritos dos estudantes em relação a tarefas referentes ao conteúdo de transformações lineares, utilizamos o referencial teórico adotado juntamente com o Quadro 1, o qual representa uma síntese das semelhanças das características dos processos do PMA de Dreyfus (2002) em relação a Tall (1995) e Resnick (1987).

Quadro 1. Síntese das semelhanças do PMA baseada em Dreyfus (2002), Tall (1995) e Resnick (1987)

\begin{tabular}{|c|c|c|c|}
\hline \multirow{5}{*}{ 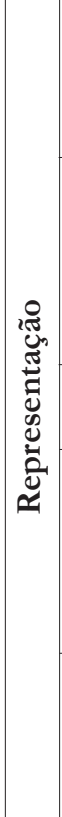 } & Simbólica (R1) & \multicolumn{2}{|c|}{$\begin{array}{l}\text { Simbolizar um objeto ou processo matemático por meio de símbolo, } \\
\text { notação ou de outra forma; e/ou } \\
\text { Manipular símbolos como se fossem objetos mentais. }\end{array}$} \\
\hline & Mental (R2) & \multicolumn{2}{|c|}{$\begin{array}{l}\text { Representar como vemos um objeto ou processo matemático entrelaçado } \\
\text { com algumas de suas propriedades; } \\
\text { Comprimir adequadamente os conhecimentos para o pequeno foco de } \\
\text { atenção, para um objeto mental. }\end{array}$} \\
\hline & Visualização (R3) & \multicolumn{2}{|c|}{ Utilizar uma imagem a fim de gerar uma representação mental; } \\
\hline & $\begin{array}{l}\text { Mudança de } \\
\text { representações } \\
\text { e alternâncias } \\
\text { entre elas (R4) }\end{array}$ & \multicolumn{2}{|c|}{$\begin{array}{l}\text { Ter várias representações de um mesmo conceito e saber utilizá-las em } \\
\text { conjunto, interligadas e, quando necessário, mudar para uma representação } \\
\text { mais eficiente (auto-regulação); e } \\
\text { Utilizar os processos de representação em um problema aplicado. }\end{array}$} \\
\hline & Modelação (R5) & \multicolumn{2}{|c|}{$\begin{array}{l}\text { Construir uma estrutura ou teoria matemática que incorpore as } \\
\text { características de um objeto ou situação física. O modelo criado também é } \\
\text { uma estrutura mental. }\end{array}$} \\
\hline \multirow{2}{*}{ 苞 } & $\begin{array}{l}\text { Generalização } \\
\text { (A1) }\end{array}$ & $\begin{array}{l}\text { Derivar ou induzir a partir de informações } \\
\text { para identificar pontos em comum e } \\
\text { expandir domínios de validade. }\end{array}$ & \multirow{2}{*}{$\begin{array}{l}\text { Novo status cognitivo: da } \\
\text { descrição para a definição; } \\
\text { Obteve o resultado a } \\
\text { partir de uma estrutura } \\
\text { em desordem aparente. }\end{array}$} \\
\hline & Sintetização (A2) & $\begin{array}{l}\text { Combinar ou compor partes de tal modo } \\
\text { a fim de formar um todo, uma entidade. }\end{array}$ & \\
\hline
\end{tabular}

Fonte: elaborado pelos autores.

\section{Procedimentos metodológicos}

As informações para a análise foram coletadas por meio da aplicação de tarefas que contemplavam o conteúdo de transformações lineares, sendo que as atividades foram realizadas por 13 estudantes do curso de Matemática de uma universidade estadual do norte do Paraná. Desses 13 estudantes, todos cursavam bacharelado em Matemática e dois desses faziam também licenciatura. 
O instrumento aplicado foi constituído por oito tarefas envolvendo o conteúdo de transformações lineares, essas tarefas foram retiradas de livros didáticos que compõem a bibliografia do curso de Matemática. E, ainda, para atingir o objetivo da pesquisa, escolhemos realizar esse estudo à luz da metodologia de análise de conteúdo segundo Bardin (2004).

Após a realização de uma leitura flutuante dos registros escritos obtidos a partir da aplicação do nosso instrumento, codificamos os registros de cada estudante utilizando a letra "E” juntamente com um número para referenciar cada um dos treze estudantes, por exemplo, o estudante de número sete ficou com o código E7. Além disso, aplicamos uma codificação para as tarefas, sendo T1, para indicar a primeira tarefa, até a tarefa de número oito, T8. Desse modo, analisamos cada tarefa olhando para um código, ou seja, “T3-E7” que significa: a tarefa três do estudante sete.

A partir disso com o corpus composto e uma primeira impressão adquirida, descrevemos todas as resoluções dos estudantes com intuito de encontrarmos semelhanças nos registros escritos de cada tarefa. Em seguida construímos os agrupamentos, relacionando elementos semelhantes do conteúdo de transformações lineares com características dos processos do PMA. E, por fim, elaboramos as categorias e a interpretação dos resultados.

\section{Análises e inferências}

Primeiramente, selecionamos os registros escritos a fim de detalhar cada um, em seguida os reescrevemos para elencar os elementos em comum e, assim, construir os agrupamentos (unidades de registro) em cada tarefa.

Após essa etapa realizamos a construção dos agrupamentos por tema, tendo como núcleo de sentido elementos relacionados com o conteúdo de transformações lineares - podendo ser símbolos, propriedades, definições ou outros -, os quais foram os indicadores para fundamentação da interpretação final.

Em seguida, analisamos os registros escritos com base em nosso referencial teórico adotado e no Quadro 1: síntese das semelhanças de características dos processos do Pensamento Matemático Avançado baseado em Dreyfus (2002), Tall (1995) e Resnick (1987), para inferirmos a respeito das características/indícios do PMA manifestados nas resoluções.

Num movimento interpretativo das análises das informações, emergiram de cada tarefa dois quadros, o primeiro referente aos agrupamentos e subagrupamentos da tarefa em questão, relacionado com o conteúdo de transformações lineares, e o segundo envolvendo os indícios/ características dos processos do PMA manifestados nos registros escritos. Esses quadros serviram como base para a construção das categorias.

Nesse artigo apresentaremos algumas partes das análises das resoluções de três tarefas do instrumento composto por oito tarefas. Escolhemos por apresentar essas três, porque a primeira diz respeito à definição de transformações lineares, a segunda por apresentar alguns exemplos de transformações, utilizando modos diferentes de representação, e a terceira por exigir que o estudante construa e aplique a matriz de uma rotação. Vejamos algumas considerações referentes às análises dessas tarefas. 
Tarefa 1: O que é uma transformação linear?

Em relação à definição de transformações lineares, quatro estudantes, E7, E8, E10, E13, manifestaram os processos de representação mental e simbólica do PMA relacionados a esse conceito, pois, utilizando símbolos para denotar elementos que compõem uma transformação linear, apresentaram a definição, mostrando que esse conceito é uma representação mental para eles, ou seja, por trás de suas notações existe o conceito formado do objeto matemático em questão.

Além desses dois processos, esses estudantes manifestaram outros processos de representação do PMA: a mudança de representações e alternância entre elas, e a modelação. Em relação aos processos de abstração, três estudantes, E7, E8, E13, apresentaram a síntese e E8 também manifestou a generalização. A seguir destacamos o registro escrito de E8 (Figura 1) que apresentou os dois processos de abstração, entre outros, de representação.

Figura 1. Registro escrito de E8 referente à definição de transformação linear

$$
\begin{aligned}
& \text { Vima transformociōo ĺnear } \\
& \text { i' quando se sotisfog as sugum. } \\
& \forall u, v \in V \text { (Vum espoco vetorid) } \\
& \text { e } \forall \alpha \in \mathbb{R} \text {, tem-s } \\
& f(u+v)=f(u)+f(v) \text {. } \\
& \alpha f(u)=f(\alpha u) \\
& \text { ou syo } \\
& V \longrightarrow V \\
& \alpha f(u+v) \longmapsto \alpha f(u)+\alpha f(v)
\end{aligned}
$$

Fonte: Resolução entregue pelo estudante.

Nesse registro escrito o estudante E8 anotou as duas condições distintas da definição e a combinação delas para ser uma transformação linear, isso explicita que manifestou mais de uma representação para um mesmo conceito e soube utilizá-las em conjunto, interligadas, mostrando que, segundo Dreyfus (2002), esse é um caminho para atingir a abstração. Assim, o estudante generalizou quando, a partir das duas condições expressas para ser uma transformação linear, identificou pontos em comum e formou apenas uma condição abrangendo as duas. Por fim, sintetizou combinando as partes de tal modo que formou a definição de transformações lineares.

O Quadro 2 apresenta uma síntese das características dos processos do PMA evidenciados nos registros escritos dos estudantes referente à tarefa 1. 
Quadro 2. Características dos processos do PMA em relação à tarefa 1

\begin{tabular}{|c|c|c|c|c|c|c|c|c|c|c|c|c|}
\hline \multicolumn{10}{|c|}{ Estudantes } \\
\hline E1 & E2 & E3 & E4 & E5 & E6 & E7 & E8 & E9 & E10 & E11 & E12 & E13 \\
\hline & & & & & & R1 & R1 & & R1 & & & R1 \\
\hline & & & & & & R2 & R2 & & R2 & & & R2 \\
\hline & & & & & & R4 & R4 & & & & & R4 \\
\hline & & & & & & R5 & R5 & & & & & R5 \\
\hline & & & & & & & A1 & & & & & \\
\hline & & & & & & A2 & A2 & & & & & A2 \\
\hline
\end{tabular}

Fonte: elaborado pelos autores.

Tarefa 2: Marque qual(is) da(s) aplicação(ões) abaixo é (são) transformação(ões) linear(es)? Justifique.

a) ( ) $T: R^{2} \rightarrow R^{2}$ definida por: $T(x, y)=(-x, y)$

b) ( ) $T: R^{2} \rightarrow R^{2}$ definida por: $T(x, y)=(x+k y, y)$

c) ( ) $\mathrm{T}: \mathrm{R}^{3} \rightarrow \mathrm{R}^{3}$ definida por: $\mathrm{T}(\mathrm{x}, \mathrm{y}, \mathrm{z})=(\mathrm{x}, \mathrm{y}, 0)$

d)( ) A rotação do $R^{2}$ no $R^{2}$.

e)( ) A translação do $R^{2}$ no $R^{2}$.

f)( ) O cisalhamento de $\mathrm{R}^{2}$ de fator $\mathrm{k}$ na direção $\mathrm{x}$.

$\mathrm{g})$ ( ) A ilustração abaixo

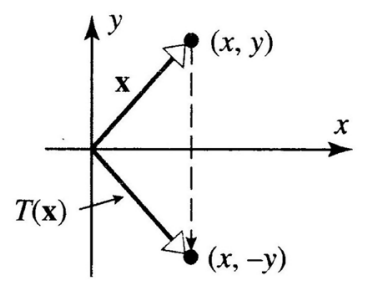

Fonte: adaptado de Anton e Rorres (2012, p. 252).

h)( ) A ilustração abaixo

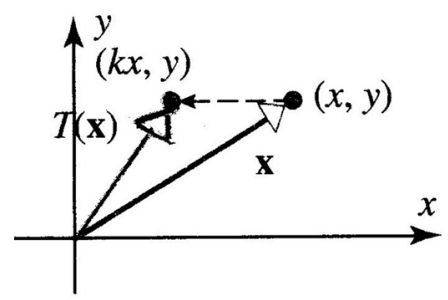

Fonte: Anton e Rorres (2012, p. 257) 
Essa tarefa pedia para que os estudantes marcassem quais itens seriam transformações lineares e justificassem suas respostas. Novamente poucos estudantes manifestaram indícios/ características dos processos do PMA e, consequentemente, poucos identificaram as transformações lineares corretas, dentre todos, somente dois estudantes acertaram.

Já os processos manifestados do PMA de representação foram: a simbólica; a mental; a visualização; e a mudança de representações e alternância entre elas, e de abstração: a síntese. Quatro estudantes, E6, E7, E10 e E13, manifestaram características desses processos, sendo que E6 e E10 manifestaram apenas a representação simbólica, E7 além da simbólica apresentou a mental, e E13 manifestou todas as citadas acima.

E13 apresenta os processos de representação simbólica e mental, pois, a partir das informações da tarefa, resolve por meio das duas propriedades da definição utilizando símbolos com suas relações a fim de provar que os itens $a, b, c$ e $h$, são transformações lineares. Além disso, manifesta o processo de visualização a partir da imagem de um item que é a da compressão de $R^{2}$ na direção de $x$ de fator $k$, ou seja, o estudante resolve a tarefa utilizando os dados do gráfico, transitando pelas representações gráficas e algébricas. Por consequência, inferimos indícios do processo de mudança de representações e alternância entre elas, porque utiliza várias representações de um mesmo conceito (DREYFUS, 2002).

Outro processo a considerar na resolução o estudante E13 é o de síntese, pois ele soube combinar relações do conteúdo a fim de formar um todo, ou seja, a demonstração correta de alguns itens. Vejamos seu registro escrito (Figura 2).

Figura 2. Registro escrito de E13 referente a aplicações de transformações lineares ${ }^{3}$

$$
\begin{aligned}
& R=(x, y) v=(a, b) \\
& t(u+v)=t(x+a, y+b)=(-x-a ; y+b)=(-x, y)+(-a, b)=+(a+t+v) \\
& +\lambda v=t(\lambda x, \lambda y)=(-\lambda x, \lambda y)=\lambda f-x, y)=\lambda+u \\
& \text { b) } t_{(u+v)}=t(x(a) y+b)=(x+c-k y+k b, y+b) \\
& =\left(x+x_{y}, y\right)+\left(a+x_{b}, b\right)=f_{(a)}+t(v) \\
& f(\lambda x, \lambda y)=\left(\lambda e^{2} k \lambda y, x y\right)=\lambda(x+k y, y)=\lambda+u
\end{aligned}
$$

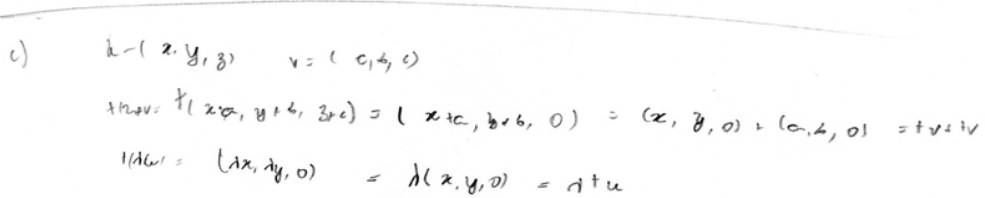

$$
\begin{aligned}
& f(x, y)+(c, b)=\left(k(x+a), \gamma^{r b}\right)=\left(k x+k_{a}, y+b\right)=(k x, y)+(k c, b) \\
& =t_{u+t v} \\
& f(t, y)=(k \lambda x, \lambda y): i(k x, y)=\lambda+u
\end{aligned}
$$

Fonte: Resolução entregue pelo estudante. 
O Quadro 3 apresenta uma síntese das características dos processos do PMA evidenciados nos registros escritos dos estudantes referente à tarefa 2.

Quadro 3. Características dos processos do PMA em relação à tarefa 2

\begin{tabular}{|c|c|c|c|c|c|c|c|c|c|c|c|c|}
\hline \multicolumn{10}{|c|}{ Estudantes } \\
\hline E1 & E2 & E3 & E4 & E5 & E6 & E7 & E8 & E9 & E10 & E11 & E12 & E13 \\
\hline & & & & & R1 & R1 & & & R1 & & & R1 \\
\hline & & & & & & R2 & & & & & & R2 \\
\hline & & & & & & & & & & & & R3 \\
\hline & & & & & & & & & & & & R4 \\
\hline & & & & & & & & & & & & A2 \\
\hline
\end{tabular}

Fonte: elaborado pelos autores.

Observamos no Quadro 3 que apenas um estudante manifestou um processo de abstração, o da síntese.

Tarefa 3: "Os operadores matriciais de $\mathrm{R}^{2}$ e $\mathrm{R}^{3}$ que movem pontos ao longo de arcos circulares são denominados operadores de rotação ou, simplesmente, rotações" (ANTON; RORRES, 2012, p. 253). As tarefas abaixo são referentes a rotações.

a) “Seja $T: R^{2} \rightarrow R^{2}$ a transformação que faz a rotação de ângulo $90^{\circ}$ no sentido anti-horário em relação à origem. Mosque que T é uma transformação linear” (POOLE, 2004, p. 191).

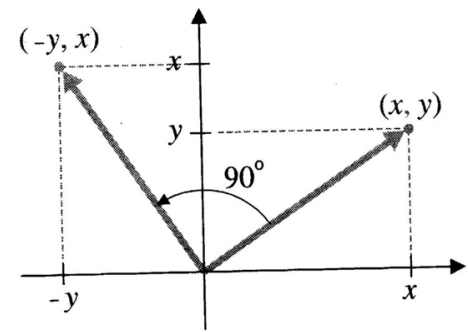

Fonte: Poole (2004, p. 191).

\footnotetext{
${ }^{3}$ Transcrição da resolução de E13 referente a tarefa 2, itens $a, b, c$ e $b$ :

" $u=(x, y) v=(a, b)$

(a) $T(u+v)=T(x+a, y+b)=(-x-a ; y+b)=(-x, y)+(-a, b)=T(u)+T(v)$

$T \lambda(u)=T(\lambda x, \lambda y)=(-\lambda x, \lambda y)=\lambda(-x, y)=\lambda T u$

(b) $T(u+v)=T(x+a, y+b)=(x+a+k y+k b, y+b)=(x+k y, y)+(a+k b, b)=T(u)+T(v)$

$T(\lambda x, \lambda y)=(\lambda x+k \lambda y, \lambda y)=\lambda(x+k \lambda, y)=\lambda T u$

(c) $u=(x, y, z) v=(a, b, c)$

$T(u+v)=T(x+a, y+b, z+c)=(x+a, y+b, 0)=(x, y, 0)+(a, b, 0)=T u+T v$

$T(\lambda u)=(\lambda x, \lambda y, 0)=\lambda(x, y, 0)=\lambda T u$

(b) $T(x, y)+(a, b)=(k(x+a), y+b)=(k x+k a, y+b)=(k x, y)+(k a, b)=T u+T v$

$T(\lambda(x, y)=(k \lambda x, \lambda y)=\lambda(k x, y)=\lambda T u$ '
} 
b) Encontre a matriz canônica de uma rotação $T: R^{2} \rightarrow R^{2}$ que move os pontos no sentido anti-horário em torno da origem por um ângulo $\theta$ (ANTON; RORRES, 2012, p. 253).

c) Utilizando o resultado do item (b), faça a rotação do ponto $(2,-1)$ de ângulo $60^{\circ} \mathrm{em}$ torno da origem a fim de obter o ponto que representa a imagem de $(2,-1)$.

Dos 13 estudantes que participaram dessa pesquisa, apenas E6, E7 e E13 responderam os três itens da tarefa 3, sendo que E10 respondeu apenas ao item (a).

Todos os processos do PMA, segundo o Quadro 1, foram manifestados nas resoluções dessa tarefa, porém somente dois estudantes, E7 e E13, apresentaram características de todos os processos; E10 manifestou a representação simbólica, a mental e a visualização; e E6, além desses processos, manifestou a mudança de representações e alternância entre elas e a generalização.

Destacamos o registro do estudante E7 (Figura 3) que apresentou características dos processos do PMA contidos no Quadro 1.

Figura 3. Registro escrito de E7 referente à tarefa de rotação

$$
\begin{aligned}
& \text { a) } T(x, y)=(-y, x) . \quad A=\left(a_{1}, a_{2}\right), B=\left(b_{1}, b_{2}\right), \alpha \in \mathbb{R} \text {. } \\
& T\left(a_{+}+\alpha\right)=T\left(a_{1}+\alpha b_{3}, a_{2}+\alpha b_{3}\right)=\left(-a_{2}+\alpha b_{2}, a_{1}+\alpha b_{1}\right)= \\
& =\left(-a_{2}, a_{1}\right)+\alpha\left(-b_{2}, b_{1}\right)=T(A)+\alpha T(B) \text {. } \\
& \text { b) Cimating; }\left[\begin{array}{cc}
\cos \theta & -\operatorname{sen} \theta \\
\operatorname{sen} \theta & \cos \theta
\end{array}\right] \\
& \text { c) }\left[\begin{array}{cc}
\cos 60^{\circ} & -\operatorname{sen} 60^{\circ} \\
\operatorname{sen} 60^{\circ} & \cos 60^{\circ}
\end{array}\right]\left[\begin{array}{c}
2 \\
-2
\end{array}\right]=\left[\begin{array}{cc}
1 / 2 & -\frac{\sqrt{3}}{2} \\
\frac{3}{2} & \frac{1}{2}
\end{array}\right]\left[\begin{array}{c}
2 \\
-\rho
\end{array}\right]=\left[\begin{array}{c}
\frac{2+\sqrt{3}}{2} \\
\frac{2 \sqrt{3}-1}{2}
\end{array}\right]
\end{aligned}
$$

Fonte: Resolução entregue pelo estudante.

O estudante E7 utiliza símbolos para representar vetores, escalares, pontos, pares ordenados e a transformação linear, juntamente com a manipulação algébrica desses símbolos para a resolução da tarefa. Assim, além da representação simbólica houve representação mental, pois em cada símbolo há um significado associado, existem esquemas internos relacionados com o conceito de rotação. 
Além disso, E7 apresenta indícios de características do processo de visualização, pois utiliza corretamente as informações da imagem a fim de gerar uma representação mental no item $a$, ou seja, "a visualização é um processo pelo qual as representações mentais podem ser geradas.” (DREYFUS, 2002, p. 31, tradução nossa).

Para o processo de mudança de representações e alternância entre elas, o estudante E7 utiliza mais de uma representação para a resolução da tarefa, por isso inferimos que usa os dados da representação gráfica, como citado acima, para a resolução dos itens, mudando de representação de pares ordenados para a representação de matriz. Desse modo, inferimos também que o estudante sabe utilizar mais de uma representação em paralelo, faz ligações entre essas representações integrando-as para resolver a tarefa em si, e utiliza a representação de forma mais eficiente, para resolver os itens separadamente, passando pelas quatro fases da representação à abstração de Dreyfus (2002).

Já para o processo de modelação que, segundo Dreyfus (2002), é manifestado quando se constrói uma estrutura ou teoria matemática que incorpore as características de um objeto ou situação física, consideramos que E7 apresentou características desse processo, pois determinou a matriz canônica da rotação e, a partir dessa, encontrou a imagem de pontos distintos em ângulos distintos. Desse modo, o estudante generalizou a matriz encontrada e usou como um modelo para determinar a rotação de um ângulo em um ponto, conforme já mencionado. Desse modo, E7, a partir de informações, derivou ou induziu a fim de identificar pontos em comum e expandir seus domínios de validade (DREYFUS, 2002).

Para a resolução do item $c$, era necessário saber como realizar a transformação do ponto pela matriz canônica, a qual é realizada pela transformação por meio de uma matriz, ou seja, revelando que, além de mostrar que uma transformação é linear utilizando as propriedades do conceito, ele soube resolver um problema aplicado utilizando outra relação de transformações lineares que é a transformação por meio de uma matriz. A partir disso, inferimos que o estudante E7 apresenta indícios do processo de síntese, pois combinou ou compôs partes a fim de formar o todo.

O Quadro 4 apresenta uma síntese das características dos processos do PMA evidenciados nos registros escritos dos estudantes referente à tarefa 3.

Quadro 4. Características dos processos do PMA em relação à tarefa 3

\begin{tabular}{|r|r|c|c|c|c|c|c|c|c|c|r|r|}
\hline \multicolumn{10}{|c|}{ Estudantes } \\
\hline E1 & E2 & E3 & E4 & E5 & E6 & E7 & E8 & E9 & E10 & E11 & E12 & E13 \\
\hline & & & & & R1 & R1 & & & R1 & & & R1 \\
\hline & & & & & R2 & R2 & & & R2 & & & R2 \\
\hline & & & & & & R3 & & & R3 & & & R3 \\
\hline & & & & & R4 & R4 & & & & & & R4 \\
\hline & & & & & & R5 & & & & & & R5 \\
\hline & & & & & A1 & A1 & & & & & & A1 \\
\hline & & & & & & A2 & & & & & & A2 \\
\hline
\end{tabular}

Fonte: elaborado pelos autores. 
A partir das análises das tarefas em relação ao conteúdo de transformações lineares, utilizando os agrupamentos referentes ao conteúdo em questão e quadros sínteses das características dos processos do PMA, construímos as categorias em relação à resolução dos estudantes, que resultam no Quadro 5.

Quadro 5. Categorias em relação aos processos do PMA manifestados pelos estudantes

\begin{tabular}{|c|l|}
\hline Estudante(s) & \multicolumn{1}{c|}{ Categorias } \\
\hline E1 e E6 & $\begin{array}{l}\text { 1. Utilizam de símbolos para representar alguns elementos da definição e } \\
\text { manipulam algebricamente, em partes, elementos de transformaços lineares. }\end{array}$ \\
\hline E10 & $\begin{array}{l}\text { 2. Utiliza de símbolos para representar alguns elementos da definição e a } \\
\text { escreve utilizando mais de uma representação em paralelo. }\end{array}$ \\
\hline E7 e E13 & $\begin{array}{l}\text { 3. Apresenta a definição de transformações lineares, utilizando símbolos } \\
\text { e manipulando-os algebricamente. Utiliza mais de uma representação em } \\
\text { paralelo para transformações lineares, combinando partes a fim de formar o } \\
\text { todo. }\end{array}$ \\
\hline $\begin{array}{l}\text { 4. Apresentam a definição de transformações lineares, utilizando símbolos } \\
\text { para representar seus elementos, manipulando-os algebricamente. Essas } \\
\text { notações mostram ter relações com os conceitos. Utilizam mais de uma } \\
\text { representação em paralelo, fazendo ligações entre essas representações, } \\
\text { integrando-as e, sempre que necessário, mudando para uma representação } \\
\text { mais eficiente. Constroem uma estrutura ou teoria, um modelo que } \\
\text { incorpora as características do objeto. Generalizam quando, a partir das } \\
\text { informações, identificam pontos em comum a fim de expandir os domínios } \\
\text { de validade. Combinam partes de tal modo a fim de formar o todo. }\end{array}$ \\
\hline
\end{tabular}

Fonte: elaborado pelos autores.

Cada categoria tem uma relação com algum(ns) processo(s) do PMA. A categoria 1 condiz ao processo de representação simbólica (R1). A categoria 2, com os processos de representação simbólica, mental e mudança de representações e alternância entre elas (R1, R2 e R4). Na categoria 3, além dos processos da categoria 2, entra também a sintetização (R1, R2, R4 e A2). E, por fim, a categoria 4 abrange todos os processos do PMA (R1, R2, R3, R4, R5, A1, e A2).

Os processos que inferimos nas categorias são resultados da análise como um todo, ou seja, se algum estudante manifestou um dos processos apenas em um item ou tarefa, pode ser que não consideremos o mesmo processo manifestado no todo.

Diante disso, evidenciamos a manifestação dos processos do PMA destacado no Quadro 5, de acordo com o Quadro 1, nas resoluções do instrumento da pesquisa. Seis estudantes manifestaram características dos processos do PMA na resolução da maioria das tarefas. 
Nos registros escritos das oito tarefas aplicadas inferimos indícios/características dos processos de representação simbólica e mental do PMA, pois, ao utilizarem símbolos, a linguagem natural, justificando suas respostas, possibilitaram-nos inferir elementos matemáticos que estão relacionados com o conceito de transformações lineares.

Além disso, em alguns registros escritos, os estudantes apresentaram características do processo de mudança de representações e alternância entre elas, ou seja, transitaram por mais de uma representação para os objetos matemáticos em questão. Para Dreyfus (2002), a utilização de mais de uma representação está ligada ao processo de aprendizagem, de modo que há uma necessidade cognitiva em questão, ou seja, o pensamento

[...] é reforçado quando os estudantes de matemática são capazes de colocar-se mentalmente em uma representação particular, por exemplo, uma visual. É ainda mais aumentada, quando eles são capazes de utilizar várias representações em paralelo. (DREYFUS, 2002, p. 39, tradução nossa)

Três estudantes (E7, E10 e E13) manifestaram o processo de modelação construindo um modelo matemático para um objeto. Para Dreyfus (2002) esse modelo pode ser usado para o estudo do comportamento do objeto, e foi o que esses estudantes fizeram, utilizando a descrição do conceito matemático, a definição de espaços transformações lineares, para a resolução das outras tarefas.

Dos seis estudantes (E1, E6, E7, E8, E10 e E13), três (E1, E6 e E8) apresentaram características apenas dos processos de representação, dois (E1 e E6) manifestando somente a representação simbólica e, o outro (E8), os processos de representação simbólica, mental e mudança de representações e alternância entre elas.

Para os processos de abstração, somente três estudantes (E7, E10, e E13) manifestaram características relativas aos dois processos que são a generalização e a síntese, englobando todo o instrumento. Segundo Dreyfus (2002), tanto a generalização quanto a síntese são incentivos para abstrair um objeto matemático. Nesse sentido, os estudantes manifestaram o processo de generalização quando, a partir de informações, encontraram pontos em comum escrevendo uma expressão geral para o objeto em questão. E, de síntese, quando combinaram elementos a respeito do objeto a fim de formar o todo, resolvendo a tarefa.

Dos processos de abstração, um estudante (E10) manifestou apenas a síntese e outros dois (E7 e E13), além da síntese, manifestaram características de generalização.

Apenas dois estudantes (E7 e E13), apresentaram características de todos os processos do PMA na resolução do instrumento todo, os quais apresentaram vários dos processos de representação e/ou abstração em cada tarefa.

Não podemos inferir se os três estudantes que manifestaram os processos de abstração do Pensamento Matemático Avançado compreendem o objeto transformações lineares, pois existem outros elementos que fazem parte desse conteúdo que não foram contemplados nesta pesquisa. Contudo, tomando como base que a compreensão para Dreyfus (2002) pode ser atingida por meio de uma sequência de atividades, nas quais processos mentais ocorrem e interagem, podemos inferir que, esses estudantes compreenderam elementos relativos a esse 
conceito, como a própria definição, pois manifestaram os processos do PMA em atividades referentes ao objeto matemático em questão.

\section{Considerações finais}

As categorias confirmam que os estudantes participantes da pesquisa podem manifestar características dos processos do Pensamento Matemático Avançado em relação a esses conceitos durante a graduação. Porém, sete estudantes não apresentaram características desses processos na resolução do instrumento, porque não responderam as tarefas e/ou escreveram respostas subjetivas que não mostram algum conhecimento do assunto. Isso é preocupante, pois todos haviam cursado a disciplina de Álgebra Linear no ano anterior.

Percebemos também nos registros escritos da maioria desses estudantes dificuldades com as notações para os conceitos, assim como a manipulação dessas notações. Entendemos que, a Álgebra Linear, a qual é uma disciplina dos primeiros anos do curso de Matemática, exige um formalismo por parte dos estudantes, com o qual eles não estão acostumados. E esse é um dos motivos pelo qual existe a necessidade dos professores oportunizarem momentos de reflexão em relação aos objetos dessa disciplina, afinal apenas definir e exemplificar conceitos / objetos matemáticos abstratos promovendo a repetição de procedimentos e técnicas não são suficientes para a compreensão.

Conforme já mencionado, o desenvolvimento do Pensamento Matemático Avançado pode contribuir para momentos de reflexão desses estudantes, a fim de que compreendam os conceitos matemáticos em questão e seus objetos envolvidos. Diante dos resultados dessa pesquisa, percebemos que conduzir os estudantes ao desenvolvimento dos processos do PMA durante o ensino e aprendizagem do conteúdo de transformações lineares pode levá-los a abstração desse objeto, ou seja, a sua compreensão (DREYFUS 2002).

Além disso, é preciso deixar claro aos estudantes quais são esses possíveis processos que estão acontecendo em seu ensino e aprendizagem e que podem potencializá-los, pois, para Dreyfus (2002), os processos do PMA não acontecem por si mesmos e, se acontecem, não são necessariamente conscientes por parte dos estudantes.

E, ainda, de acordo com Dreyfus (2002), um dos objetivos do professor de matemática do Ensino Superior é tornar os estudantes conscientes dos processos envolvidos no PMA e suas interações, pois, desse modo, é possível potencializar o pensamento matemático do estudante próximo ao de um matemático. Portanto, promover conscientemente o desenvolvimento desse tipo de pensamento nos estudantes, possibilita o caminho à abstração do objeto matemático em questão.

Nesse sentido, percebemos que existem dificuldades por parte dos estudantes quanto à compreensão dos objetos matemáticos de um conceito abstrato como transformações lineares. Por esse motivo, há a necessidade de que o professor do Ensino Superior possibilite aos seus alunos meios para suprirem essas dificuldades, e que busquem "novas" formas/metodologias de ensino e aprendizagem além da tradicional, que é definir e exemplificar o objeto, para que esses conceitos abstratos sejam realmente compreendidos. 


\section{Referências}

ANTON, H.; RORRES, C. Álgebra linear com aplicações. 10. ed. Porto Alegre: Bookman, 2012.

BARDIN, L. Análise de conteúdo. 3. ed. Lisboa: Edições 70, 2004.

DOMINGOS, A. Teorias cognitivas e aprendizagem de conceitos matemáticos avançados. In: SEMINÁRIO DE INVESTIGAÇÃO EM EDUCAÇÃO MATEMÁTICA, 17., 2006, Setúbal. Actas...Setúbal: Associação de Professores de Matemática, 2006. p. 51-81.

DREYFUS, T. Advanced mathematical thinking processes. In: TALL, D. Advanced mathematical thinking. Dordrecht: Kluwer, 2002. p. 25-41.

FIORENTINI, D.; MIORIN, M.; MIGUEL, A. Contribuição para um repensar... a educação algébrica elementar. Pro-Posições, Campinas, v. 4, n. 1, p. 78-91, 1993. Disponível em: <http://www.proposicoes.fe.unicamp.br/proposicoes/textos/10-artigos-fiorentinid_etal. pdf>. Acesso em: 25 jan. 2016.

POOLE, D. Álgebra linear. São Paulo: Pioneira Thomson Learning, 2004.

RESNICK, L. B. Education and learning to think. Washington: National Academy Press, 1987.

TALL, D. Cognitive growth in elementary and advanced mathematical thinking. In: MEIRA, L.; CARRAHER, D. (Ed.). Proceedings of 19th International Conference for the Psychology of Mathematics Education. Recife: UFPE, 1995. v. 1, p. 61-75.

TALL, D. The psychology of advanced mathematical thinking. In: . Advanced mathematical thinking. Dordrecht: Kluwer, 2002. p. 3-21.

Artigo recebido em 20/08/2014. Aceito em 25/09/2015. 